\title{
Prognostic factors and survival in endocrine tumor patients: comparison between gastrointestinal and pancreatic localization
}

\author{
Francesco Panzuto ${ }^{1}$, Silvia Nasoni ${ }^{1}$, Massimo Falconi ${ }^{4}$, Vito Domenico Corleto ${ }^{1}$, \\ Gabriele Capurso ${ }^{1}$, Sara Cassetta ${ }^{1}$, Michela Di Fonzo ${ }^{1}$, Valentina Tornatore ${ }^{1}$, \\ Massimo Milione ${ }^{1,5}$, Stefano Angeletti ${ }^{1}$, Maria Sofia Cattaruzza ${ }^{2}$, \\ Vincenzo Ziparo ${ }^{3}$, Cesare Bordi ${ }^{5}$, Paolo Pederzoli ${ }^{4}$ and Gianfranco Delle Fave ${ }^{1}$
}

Departments of ${ }^{1}$ Digestive and Liver Disease, ${ }^{2}$ Public Health and ${ }^{3}$ Surgery, II School of Medicine, University 'La Sapienza', Ospedale Sant'Andrea, via di Grottarossa 1035, 00189, Roma, Italy

${ }^{4}$ Department of Surgery, University of Verona, 37100 Italy

${ }^{5}$ Department of Pathology and Laboratory Medicine, University of Parma, 43100 Italy

(Requests for offprints should be addressed to G Delle Fave; email: gianfranco.dellefave@uniroma1.it)

\begin{abstract}
Since gastro-entero-pancreatic endocrine tumors are rare and heterogeneous diseases, their prognosis and long-term survival are not well known. This study aimed at identifying prognostic factors and assessing long-term survival in gastro-entero-pancreatic endocrine tumors. A total of 156 patients enrolled. Prognostic factors were determined by univariate/multivariate analysis; survival rates were assessed by the Kaplan-Meier method. The tumors were non-functioning in $59.6 \%$ of patients, and originated from the pancreas in $42.9 \%$. At diagnosis, $64.3 \%$ of patients had metastases. The tumors were well differentiated in $89.6 \%$ of patients. Ki67 was $>2 \%$ in $39.6 \%$ of patients. Primary tumor size was $>3 \mathrm{~cm}$ in $49.6 \%$ of cases studied. For the univariate analysis, the negative prognostic factors were: pancreatic origin (rate ratio 4.64, $P=0.0002$ ), poorly differentiated tumor (rate ratio $7.70, P=0.0001$ ), primary tumor size $>3 \mathrm{~cm}$ (rate ratio $4.26, P=0.0009$ ), presence of distant metastases (liver: rate ratio 5.88, $P=0.01$; distant extra-hepatic: rate ratio 13.41, $P=0.0008$ ). The pancreatic site, the poor degree of differentiation and the distant metastases were confirmed as negative prognostic factors at multivariate analysis. Overall 5-year survival rate was $77.5 \%$. Survival rates differed according to: primary tumor site $(62 \%$ for pancreatic vs $89.9 \%$ for gastrointestinal tract, $P=0.0001)$ and size $(65.7 \%$ for $>3 \mathrm{~cm}$ vs $88.8 \%$ for $\leq 3 \mathrm{~cm}, P=0.0003)$, degree of differentiation (22\% for poor vs $86.8 \%$ for good, $P<0.0001)$, Ki67 (53.5\% for $>2 \%$ vs $90.1 \%$ for $\leq 2 \%, P=0.003$ ), metastases $(96.1,77,73.3$ and $50.1 \%$ for absent, local, liver and distant extra-hepatic metastases respectively), age at diagnosis $(85.3 \%$ for $\leq 50$ years vs $70.3 \%$ for $>50$ years, $P=0.03$ ). Although $64.3 \%$ of gastro-entero-pancreatic endocrine tumors present metastases at diagnosis, the 5-year survival rate is $77.5 \%$. Pancreatic site, a poor degree of tumor cell differentiation and distant extra-hepatic metastases are the major negative prognostic factors.
\end{abstract}

Endocrine-Related Cancer (2005) 12 1083-1092

\section{Introduction}

Gastro-entero-pancreatic endocrine tumors (GEP ETs) are rare neoplasms, with $1-4$ cases per 100000 people per year (Quaedvlieg et al. 2001, Modlin et al. 2003, Lepage et al. 2004, Taal \& Visser 2004). Usually, they are considered less aggressive, when compared with exocrine carcinomas, on account of their slow growth and also the long survival observed in patients with advanced disease; overall 5-year survival rates range from 38 to $100 \%$, depending on tumor site and staging (Modlin et al. 2003). However, GEP ETs 
belong to a heterogeneous family of tumors, as they may originate from the pancreas (pancreatic endocrine tumors (PETs)) or gastrointestinal (GI) tract (GI carcinoids), and may be associated with a specific syndrome due to the uncontrolled hormone secretion; this differentiates the so-called 'functioning tumors' from the 'non-functioning' in which the clinical picture is only related to the mass-effect derived from tumor growth (Barakat et al. 2004). Furthermore, several other elements characterize the specific kinds of GEP ETs: histological/immunohistochemical features; possible association with multiendocrine syndrome type I (MEN-I); and expression of somatostatin receptor subtypes (sstr). Thus, due to their rarity, and clinical and biological heterogeneity, the identification of prognostic factors and assessment of long-term survival are difficult. Furthermore, despite the increasing interest in GEP ETs over the last few years, few studies have been addressed at evaluating series of patients including both 'functioning' and 'non-functioning' tumors that originated either in the GI tract or in the pancreas. Although some reports have prospectively investigated the clinical course and the survival of patients with gastrinoma ( $\mathrm{Yu}$ et al. 1999, Weber et al. 1995), the majority of the studies including other kinds of GEP ETs refer to retrospective studies (Burke et al. 1997, Madeira et al. 1998, Phan et al. 1998, Shebani et al. 1999, Quaedvlieg et al. 2001, Chu et al. 2002, Hochwald et al. 2002, Gullo et al. 2003, Modlin et al. 2003, Pape et al. 2004), some of them evaluating data reported from national cancer registries (Shebani et al. 1999, Quaedvlieg et al. 2001, Modlin et al. 2003). Moreover, the majority of the published studies have enrolled only patients with either PETs (Phan et al. 1998, Hochwald et al. 2002, Gullo et al. 2003, Gibril \& Jensen 2004) or GI carcinoids (Burke et al. 1997, Shebani et al. 1999, Soreide et al. 2000), both kinds of tumors have been rarely investigated in the same study (Madeira et al. 1998, Johanson et al. 1999, Pape et al. 2004). Thus, to the best of our knowledge, there is a lack of prospective data comparing prognostic factors and survival rates of gastrointestinal and pancreatic endocrine tumors. This prospective study aimed, therefore, to assess the impact of clinical and patho-biological variables on long-term survival in a consecutive series of patients with PETs or GI carcinoids.

\section{Patients and methods}

\section{Study design}

All consecutive patients with PETs or GI carcinoids, seen in the Department of Digestive and Liver Disease of the University 'La Sapienza', Roma between 1983 and 2003, were included in this prospective study, as a result of the National Cooperative Programme on Digestive Endocrine Tumors established together with the Pathology Unit of the University of Parma and the Endocrine Surgery Unit of the University of Verona. The design of this programme included: a baseline visit, during which personal data, lifestyle, past medical history (including previous biochemical or instrumental examinations) and symptoms were collected in a structured questionnaire; and subsequent follow-up visits which were scheduled at 6-month intervals. A blood sample was taken on each of these occasions to assess tumor markers, as previously described (Panzuto et al. 2004). The diagnosis of endocrine tumors was confirmed by an expert pathologist (CB) upon conventional histological and immunohistochemical examinations (chromogranin A, synaptophysin, neuron-specific enolase and staining with specific peptides - according to tumor secretion) of surgical specimens (or biopsy samples of the primary tumor or liver metastases, if the patients had not undergone surgery) in all but seven patients. These seven had Zollinger-Ellison syndrome (ZES), and, due to the absence of detectable tumor lesions, histological examination was not feasible; therefore diagnosis of gastrinoma was based, as previously reported, on assessment of serum gastrin samples (basal and secretin provocative test), and on measurement of basal and maximal acid output after pentagastrin injection (Frucht et al. 1989, Fishbeyn et al. 1993). Tumor staging was performed by conventional imaging procedures, including computed tomography (CT) (helical technology was used after 1999 (Panzuto et al. 2003)), ultrasonography and somatostatin receptor scintigraphy (SRS) (after 1992 (Corleto et al. 1996)). Tumor staging was repeated yearly, or at shorter intervals if deemed necessary following assessment of clinical or biochemical markers. At each visit, the physicians collected all the medical data which were then stored into the database; the database was updated at each subsequent visit. Data on previous/current treatments were also recorded. Findings on medical treatment were not, however, considered in the final analysis due to variations in the procedures, drugs and route of administration that occurred during the long period over which the present study was performed. The prognostic significance of the following variables was assessed: sex, age, association with specific clinical syndromes, primary tumor site and size, degree of differentiation of tumor cells, Ki67 value on tumor tissue, presence of metastases (lymph nodes, liver, distant extra-hepatic), sstr expression (assessed by SRS). 


\section{Tumor definitions}

The tumors were defined as being without an associated syndrome (non-functioning) if no specific symptoms/signs were induced by tumor hormone overproduction; otherwise they were defined as having an associated syndrome (functioning) As far as the tumor extension was concerned, patients were classified - according to imaging procedures results and/or surgical findings - as follows: group I, where only the primary tumor was present; group II, in the presence of local lymph-node metastases; group III, if there were liver metastases; group IV, in the presence of distant extra-hepatic metastases. Resection of the tumor was defined as radical if tumor lesions were completely removed by the surgeon (or endoscopist, in the case of polyps amenable to endoscopic excision) and the excision margins were negative for neoplastic infiltration, upon histological examination.

\section{Statistical analysis}

The main outcome variable was survival from the date of diagnosis to the last date of follow-up or death. Actuarial survival probabilities were calculated by the Kaplan-Meier method and comparisons within variables were made using the log rank test. Univariate analysis was performed to evaluate the crude effects of prognostic factors on survival. Multivariate analysis was performed using the Cox proportional hazards model. The strategy used to determine which variables should be included in the multivariate analysis was based on whether the variable was of direct interest or merely a confounder. Variables of interest were included, while confounders that showed little or no effect were eliminated. After the elimination of these confounders, factors of interest were eliminated if their effect was not statistically significant at the 5\% level. This strategy was considered more efficient in the search for true prognosis variables than an automatic step-up or step-down elimination procedure. The two-tailed Fisher's exact test was used to compare percentages in the different subgroups. A $P$ value of $<0.05$ was considered statistically significant. The patients with MEN-I were not included in the univariate and multivariate analysis, due to the presence of multiple primary tumors arising from different sites. Moreover, while the site of primary tumor (GI carcinoids or PETs) was evaluated as a variable both in univariate and multivariate analysis, given the relative small number of patients it was not feasible to carry out the multivariate analysis of risk factors separately on the two subgroups.

\section{Results}

A total of 185 patients ( 92 male and 93 female, median age 51 years, range 10-78 years) with a diagnosis of endocrine tumor were evaluated. Of these, 29 were excluded from the final analysis due to the lack of essential data in the database $(n=16)$, or because the primary tumor was not located in the gastro-enteropancreatic system. Thus, 156 patients were enrolled: they included 74 males (median age 54 years, range 10-78 years) and 82 females (median age 49 years, range 15-78 years), who were observed during a median follow-up of 42.5 months (range 3-256 months). Of these patients, $93(59.6 \%)$ had no associated syndrome (non-functioning tumors) and $16(10.3 \%)$ had MEN-I. Overall, in $73(46.8 \%)$ and $67(42.9 \%)$ patients, the tumor was localized in the GI tract (GI carcinoids) or in the pancreas (PETs) respectively. In the remaining $16(10.3 \%)$ patients, the site of the primary tumor was unknown (Table 1). At diagnosis, $99(64.3 \%)$ patients had metastases: in 18 patients $(11.7 \%)$ these were in the loco-regional lymph node only (group II); in 61 patients $(39.6 \%)$ they were in the liver (group III); and in 20 patients $(13 \%)$ (group IV), distant extra-hepatic metastases were present (bone in 8 patients, lung in 8 and peritoneum in 6). Data on disease staging were not available in the remaining 2 patients. In the majority of the patients in the present study, the tumor showed a

Table 1 General features of the patients

\begin{tabular}{lccc}
\hline Primary site & $\begin{array}{c}\text { Overall }(\boldsymbol{n}=\mathbf{1 5 6}) \\
\boldsymbol{n}(\%)\end{array}$ & $\begin{array}{c}\text { With syndrome } \dagger(\boldsymbol{n}=\mathbf{6 3}) \\
\boldsymbol{n}(\%)\end{array}$ & $\begin{array}{c}\text { Without syndrome }(\boldsymbol{n}=\mathbf{9 3}) \\
\boldsymbol{n}(\%)\end{array}$ \\
\hline PETs & $67(42.9)$ & $26(38.8)$ & $41(61.2)$ \\
Gl carcinoids* & $73(46.8)$ & $24(32.9)$ & $49(67.1)$ \\
Unknown & $16(10.3)$ & $13(81.2)$ & $3(18.8)$ \\
\hline
\end{tabular}

*Jejunum-ileum $(n=42)$, rectum $(n=13)$, appendix $(n=8)$, duodenum $(n=6)$, stomach $(n=3$, all sporadic type III carcinoid), colon $(n=1)$.

†Zollinger-Ellison syndrome $(n=30)$, carcinoid syndrome $(n=27)$, glucagonoma $(n=3)$, somatostatinoma $(n=1)$, Vasoactive Intestinal Polypeptide releasing tumor $(n=1)$, malignant insulinoma $(n=1)$. 
Table 2 General features of patient population. Values in the square brackets refer to the number of patients in whom the data was available

\begin{tabular}{|c|c|c|c|c|c|c|}
\hline & $\begin{array}{l}\text { Overall } \\
n(\%)\end{array}$ & $\begin{array}{c}\text { Pancreas } \\
n(\%)\end{array}$ & $\begin{array}{c}\text { GI tract } \\
n(\%)\end{array}$ & $\begin{array}{c}\text { Unknown } \\
n(\%)\end{array}$ & $\begin{array}{c}\text { With syndrome } \\
n(\%)\end{array}$ & $\begin{array}{c}\text { Without syndrome } \\
n(\%)\end{array}$ \\
\hline \multicolumn{7}{|c|}{ Disease staging $[n=154]$} \\
\hline Group I & $55(35.7)$ & $20(36.4)$ & $28(50.9)$ & $7(12.7)$ & $19(34.6) \ddagger$ & $36(65.4)$ \\
\hline Group II & $18(11.7)$ & $6(33.3)$ & $12(66.7)$ & - & $4(22.2) \ddagger$ & $14(77.8)$ \\
\hline Group III & $61(39.6)$ & $28(45.9)$ & $26(42.6)$ & $7(11.5)$ & $31(50.8)$ & $30(49.2)$ \\
\hline Group IV & $20(13)$ & $11(55)$ & $7(35)$ & $2(10)$ & $8(40)$ & $12(60)$ \\
\hline \multicolumn{7}{|c|}{ Differentiation of tumor cells $[n=125]$} \\
\hline Good & $112(89.6)$ & $49(43.7)$ & $56(50)$ & 7 (16.3) & $42(37.5)^{\wedge}$ & $70(62.5)$ \\
\hline Poor & $13(10.4)$ & $9(69.3)^{\star}$ & $3(23)$ & $1(7.7)$ & - & $13(100)$ \\
\hline \multicolumn{7}{|c|}{$\mathrm{Ki67}[n=96]$} \\
\hline$\leq 2 \%$ & $58(60.4)$ & $22(37.9)$ & $30(51.7)$ & $6(10.4)$ & $24(41.4)$ & $34(58.6)$ \\
\hline$>2 \%$ & $38(39.6)$ & $27(71) \dagger$ & $9(23.7)$ & $2(5.3)$ & $6(15.8) \S$ & $32(84.2)$ \\
\hline \multicolumn{7}{|c|}{ Primary tumor size $[n=129]$} \\
\hline$\leq 3 \mathrm{~cm}$ & $65(50.4)$ & $17(26.2) \dagger$ & $48(73.8)$ & - & $26(40)$ & $39(60)$ \\
\hline$>3 \mathrm{~cm}$ & $64(49.6)$ & $46(71.9) \dagger$ & $18(28.1)$ & - & $17(26.6) \S$ & $47(73.4)$ \\
\hline \multicolumn{7}{|c|}{ SRS $[n=105]$} \\
\hline Positive & $97(92.4)$ & $51(52.6)$ & $38(39.2)$ & $8(8.2)$ & $47(48.5)$ & $50(51.5)$ \\
\hline Negative & $8(7.6)$ & $6(75)$ & $2(25)$ & - & $1(12.5)^{\circ}$ & 7 (87.5) \\
\hline
\end{tabular}

Group I: no metastases; Group II: only local lymph-node metastases; Group III: liver metastases present; Group IV: distant extrahepatic metastases present. GI = gastrointestinal; SRS = somatostatin receptor scintigraphy.

${ }^{*} p=0.02, \dagger p<0.0001$ vs Gl tract.

$\ddagger p=0.002,{ }^{\wedge} p=0.0002, \S p<0.0001, \# p=0.03,{ }^{\circ} p=0.01$ vs tumors without syndrome.

good degree of differentiation $(89.6 \%)$. In all but 8 patients $(92.4 \%)$, the tumor expressed sstr, as shown by a positive octreoscan (Table 2 ).

\section{Univariate and multivariate analysis}

The following variables were significantly associated with negative outcome: age $>50$ years, primary tumor site (pancreatic) and size $(>3 \mathrm{~cm}), \mathrm{Ki} 67$ on tumor cells $>2 \%$, degree of differentiation (poorly differentiated tumors), presence of metastases at time of diagnosis (hepatic and distant extra-hepatic). However, at multivariate analysis, the pancreatic site of the primary tumor, the poor degree of differentiation and the presence of distant extra-hepatic metastases were confirmed as significant (Table 3). Similar findings were obtained when the subgroup of patients with appendiceal tumors was excluded from the risk factors analysis; it is well known that these tumors are usually characterized by a low risk of metastases and a relatively benign behavior (Sutton et al. 2003). In fact, even in this subanalysis, the same risk factors were identified by multivariate analysis (pancreatic site, rate ratio $3.66(P=0.01)$; poor degree of differentiation, rate ratio $3.00(P=0.03)$; distant extra-hepatic metastases, rate ratio $5.64(P=0.03))$. When the risk factor analysis was performed separately on GI carcinoids and PETs, poor degree of differentiation of tumor cells and the presence of distant extra-hepatic metastases were confirmed as significant in the subgroup of patients with PETs. On the contrary, no statistically significant risk factor was identified in the subgroup of GI carcinoids (Table 4).

\section{Survival}

A total of 31 patients $(19.9 \%), 15$ males and 16 females, died of disease during follow-up. Death occurred at a median time interval after diagnosis of 18 months (range 3-108 months). Of these, 17 patients $(54.8 \%)$ had no associated syndrome and $23(74.2 \%)$ had a pancreatic tumor. Furthermore, 25 patients $(80.6 \%)$ had advanced disease at the time of diagnosis (groups III and IV). Specifically, 10 patients were in group IV, 15 in group III, 3 in group II, and 2 in group I. The remaining patient had a biochemical diagnosis of ZES without visible lesions, and died due to severe bleeding from peptic ulcers. Overall, the 5-year survival rate was $77.5 \%$. Statistically significant different survival curves were observed with respect to patient's age at time of diagnosis, primary tumor site and size, degree of tumor differentiation, 
Table 3 Univariate and multivariate analysis

\begin{tabular}{|c|c|c|c|c|c|c|}
\hline \multirow[b]{2}{*}{ Variable } & \multicolumn{3}{|c|}{ Univariate } & \multicolumn{3}{|c|}{ Multivariate } \\
\hline & Rate ratio & $95 \% \mathrm{Cl}$ & $p$ & Rate ratio & $95 \% \mathrm{Cl}$ & $p$ \\
\hline Male sex (Yes vs No) & 0.92 & $0.45-1.88$ & 0.815 & & & \\
\hline Age $>50$ yrs (Yes vs No) & 2.33 & $1.06-5.12$ & 0.035 & & & \\
\hline Functional status (without vs with syndrome) & 1.03 & $0.50-2.13$ & 0.937 & & & \\
\hline Primary tumor site (pancreas vs GI tract) & 4.64 & $2.06-10.45$ & 0.0002 & 3.69 & $1.26-10.81$ & 0.017 \\
\hline Primary tumor size $(>3 \mathrm{~cm}$ vs $\leq 3 \mathrm{~cm})$ & 4.26 & $1.81-10.03$ & 0.0009 & & & \\
\hline Tumor degree of differentiation (poor vs good) & 7.70 & $3.11-19.02$ & 0.0001 & 2.99 & $1.09-8.20$ & 0.034 \\
\hline Ki 67 value $(>2 \%$ vs $\leq 2 \%)$ & 3.84 & $1.35-10.97$ & 0.011 & & & \\
\hline \multicolumn{7}{|l|}{ Disease extension* (vs group I) } \\
\hline Group II & 4.20 & $0.70-25.29$ & 0.116 & 2.57 & $0.35-19.00$ & 0.353 \\
\hline Group III & 5.88 & $1.34-25.75$ & 0.018 & 3.26 & $0.70-15.24$ & 0.132 \\
\hline Group IV & 13.41 & $2.93-61.37$ & 0.0008 & 5.81 & $1.12-30.10$ & 0.036 \\
\hline SRS (positive vs negative) & 0.53 & $0.16-1.78$ & 0.302 & & & \\
\hline
\end{tabular}

*Group I: no metastases; Group II: only local lymph-node metastases; Group III: liver metastases present; Group IV: distant extrahepatic metastases present. $\mathrm{Gl}=$ gastrointestinal; SRS = somatostatin receptor scintigraphy.

Table 4 Univariate analysis in PETs and GI carcinoids

\begin{tabular}{|c|c|c|c|c|c|c|}
\hline \multirow[b]{2}{*}{ Variable } & \multicolumn{3}{|c|}{ PETs } & \multicolumn{3}{|c|}{ GI carcinoids } \\
\hline & Rate ratio & $95 \% \mathrm{Cl}$ & $p$ & Rate ratio & $95 \% \mathrm{Cl}$ & $p$ \\
\hline Male sex (Yes vs No) & 0.58 & $0.24-1.37$ & 0.213 & 2.29 & $0.42-12.51$ & 0.339 \\
\hline Age $>50$ yrs (Yes vs No) & 1.59 & $0.66-3.87$ & 0.301 & 5.62 & $0.65-48.25$ & 0.115 \\
\hline Functional status (without vs with syndrome) & 0.90 & $0.38-2.15$ & 0.811 & 0.38 & $0.07-2.11$ & 0.269 \\
\hline Primary tumor size $(>3 \mathrm{~cm}$ vs $\leq 3 \mathrm{~cm})$ & 3.40 & $0.79-14.71$ & 0.101 & 2.58 & $0.52-12.81$ & 0.246 \\
\hline Tumor degree of differentiation (poor vs good) & 3.43 & $1.22-9.67$ & 0.019 & NA & NA & NA \\
\hline Ki 67 value $(>2 \%$ vs $\leq 2 \%)$ & 1.69 & $0.54-5.33$ & 0.371 & 4.12 & $0.26-66.32$ & 0.318 \\
\hline \multicolumn{7}{|l|}{ Disease extension* (vs group I-II) } \\
\hline Group III & 5.43 & $1.21-24.32$ & 0.027 & $3.70 \dagger$ & $0.43-31.69$ & 0.232 \\
\hline Group IV & 11.73 & $2.48-55.60$ & 0.001 & & & \\
\hline SRS (positive vs negative) & 0.65 & $0.19-2.27$ & 0.503 & NA & NA & NA \\
\hline
\end{tabular}

*Group I: no metastases; Group II: only local lymph-node metastases; Group III: liver metastases present; Group IV: distant extrahepatic metastases present. SRS = somatostatin receptor scintigraphy. NA = not applicable. †Group III or IV vs Group I-II.

proliferation as assessed by Ki67 index on tumor tissue, and disease extension (Table 5). Moreover, statistically different survival rates $(P=0.0001)$ were observed between PETs and GI carcinoids even when the appendiceal tumors were not considered in this latter group, 5-year survival rates being 62 and $89.2 \%$ respectively. In contrast, the 5-year survival rate was not significantly related to sex (male $77.8 \%$ vs female $77 \%$ ), association with specific syndrome (with syndrome $79.6 \%$ vs without syndrome $75.7 \%$ ) and sstr expression (present $73 \%$ vs absent $45.7 \%$ ). Furthermore, no difference was observed when survival was calculated for PETs or GI carcinoids, according to the functional status (non functioning vs functioning PETs, 58.5 vs $66.6 \%$ respectively; non-functioning vs functioning GI carcinoids, 94.3 vs $86.6 \%$ respectively).

\section{Treatment of patients}

Treatment data were available in 139 patients. Of these, $28(20.1 \%)$ were not suitable for surgery due to advanced disease, and received medical treatment only; 15 patients $(10.8 \%)$ did not receive any treatment, due to the presence of ZES which required treatment with proton pump inhibitors only $(n=12)$ or because the patient refused therapy $(n=3)$. The remaining 96 patients $(69.1 \%)$ underwent surgical procedure, which was considered radical in 57 of the cases $(59.3 \%)$ ). Of these, 15 patients $(26.3 \%)$ relapsed after a median interval of 13 months (range 3-84 months) after radical surgery. Two patients were lost at early follow-up after radical surgery. The remaining 40 patients $(70.2 \%)$ were considered cured by surgery, due to the absence 
Table 5 Survival of patients

\begin{tabular}{|c|c|c|c|}
\hline & 3 years survival rate (\%) & 5 years survival rate (\%) & p (log-rank test) \\
\hline \multicolumn{4}{|c|}{ Age at diagnosis } \\
\hline$>50$ years & 74.7 & 73.3 & \multirow[t]{2}{*}{0.03} \\
\hline$\leq 50$ years & 89.4 & 85.3 & \\
\hline \multicolumn{4}{|c|}{ Primary tumor site } \\
\hline PETs & 71.3 & 62 & \multirow[t]{2}{*}{0.0001} \\
\hline GI carcnoids & 89.9 & 89.9 & \\
\hline \multicolumn{4}{|c|}{ Primary tumor size } \\
\hline$>3 \mathrm{~cm}$ & 71.4 & 65.7 & \multirow[t]{2}{*}{0.0003} \\
\hline$\leq 3 \mathrm{~cm}$ & 90.7 & 88.8 & \\
\hline \multicolumn{4}{|c|}{ Tumor degree of differentiation } \\
\hline Poor & 44 & 22 & \multirow[t]{2}{*}{$<0.0001$} \\
\hline Good & 89.7 & 86.8 & \\
\hline \multicolumn{4}{|l|}{ Ki67 value } \\
\hline$>2$ & 73.6 & 53.5 & \multirow[t]{2}{*}{0.003} \\
\hline$\leq 2$ & 90.7 & 90.1 & \\
\hline \multicolumn{4}{|c|}{ Disease extension } \\
\hline Stage I & 96.1 & 96.1 & \multirow[t]{4}{*}{$0.04^{*}$} \\
\hline Stage II & 77 & 77 & \\
\hline Stage III & 78.5 & 73.3 & \\
\hline Stage IV & 65 & 50.1 & \\
\hline
\end{tabular}

*Stage IV vs Stage III.

of residual disease at imaging controls, the normalization of tumor marker levels and the absence of specific symptoms in 'functioning tumors'; this assessment was made during a median post-operative follow-up period of 57 months (in these patients, subsequent medical treatment was not administered). Thus, surgery was considered curative in 40 out of 96 operated patients $(41.7 \%)$. A proportion of $76.8 \%(n=63)$ of the patients with visible tumor lesions $(n=82$, comprising: patients not suitable for surgery $(n=28)$, patients undergoing non-radical surgery $(n=39)$, or patients with disease recurrence after radical surgery $(n=15))$ showed a progressive disease during followup, according to WHO criteria. Of these tumors, 39 $(61.9 \%)$ were located in the pancreas and 21 in the GI tract $(33.3 \%)(P=0.002)$. In the remaining three patients $(4.8 \%)$, the primary tumor site was unknown. As far as the functional status is concerned, 36 progressive tumors $(57.1 \%)$ were not associated with a specific syndrome. Medical treatment was generally based on somatostatin analogs, which were administered in 81 out of 84 patients $(96.4 \%)$ who underwent medical therapy - in 19 of these patients in association with $\alpha$-interferon. Systemic chemotherapy was performed in 16 patients. In nine patients, liver metastases were also treated by chemoembolization and/or radiofrequency ablation. Furthermore, eight patients received radiolabelled somatostatin analog therapy.

\section{Discussion}

Knowledge of the prognostic factors and long-term survival rates in patients with GEP ETs represents a crucial point in the management of these diseases, which, as is well known, are characterized by a particular clinical and biological heterogeneity with substantial differences in terms of phenotype and relative behavior (Rindi \& Bordi 2003). The first finding of importance in the present study is that $64.3 \%$ of patients already had metastases at the time of diagnosis, irrespective of the site of the primary tumor. No difference, in fact, was observed between PETs and GI carcinoids with regard to the disease extension (Table 2). However, a relatively higher, although not significant, prevalence of more advanced disease (group III to IV) was observed in PETs, thus suggesting that in this group of patients diagnosis is usually late, probably due to the lack of specific symptoms $(61.2 \%$ of PETs had no associated syndrome). Despite the high prevalence of metastatic disease at the time of diagnosis, the overall 5-year survival rate was $77.5 \%$, thus confirming that GEP ETs can be considered slow-growing tumors with relatively low aggressive behavior. The pancreatic site represents an independent variable predictive of an unfavorable outcome (rate ratio $=3.69, P=0.01$ at multivariate analysis), with long-term survival in this 
subgroup of patients being significantly worse when compared with GI carcinoids ( $62 \%$ vs $89.9 \%$ at 5 years respectively, $P<0.0001$ ) (Tables 2 and 5). In fact, very few data are available on the comparison of the survival of endocrine tumors in relationship to their site (GI tract or pancreas). Johanson et al. (1999) compared the two subgroups of patients, however including, in their retrospective analysis, a smaller number of patients (64 midgut carcinoids and 25 PETs), all submitted to surgical treatment. The survival rate observed in our series, for PETs, is in agreement with other studies (Rigaud et al. 2001, Gullo et al. 2003), in which a survival of $50-70 \%$ was reported. However, it is higher than the data reported by Modlin et al. (2003) in a retrospective analysis of a cancer registry referring to the last three decades; and also data reported by Kent et al. (1981), Chu et al. (2002) and Lepage et al. (2004) who reported a survival rate of $30-40 \%$. This discrepancy is probably due not only to the different features of the populations studied, which included mostly patients with advanced disease, but also to the different design of the studies, generally retrospective (Modlin et al. 2003, Lepage et al. 2004, Chu et al. 2002, Gullo et al. 2003). In the present study, the negative impact of the pancreatic primary tumor localization may also be related to the association with other negative prognostic factors, such as poor tumor cell differentiation, high Ki67 value and larger tumor size (Tables 2 and 4). The highest rate ratio, for negative outcome, was observed when distant extra-hepatic lesions were present (group IV: rate ratio $5.81, P=0.03$ at multivariate analysis) (Tables 2 and 5). This finding highlights the critical role of distant non-hepatic metastases, such as bone and lung lesions, the presence of which has rarely been taken into consideration in other studies concerning the survival rates associated with GEP ETs (Madeira et al. 1998); in the present investigation, these distant non-hepatic metastases have been demonstrated to be able to identify a specific subgroup of patients with a worse prognosis and shorter survival. Thus, these lesions should always be sought during the initial staging of the disease as well as during follow-up, by means of accurate staging which includes both SRS and helical CT (Panzuto et al. 2003), to identify those patients in whom short-interval follow-up and a more aggressive therapeutic approach are mandatory. As far as the 'functional status' is concerned, no difference in survival was observed between the patients with or without an associated syndrome, even when the role of this variable was separately analyzed in the two subgroups of PETs and GI carcinoids. At present, much controversy exists concerning the possible role of the functional status as a prognostic factor. In fact, our data are not in agreement with those of other authors, who reported a less favorable survival in patients with no associated syndrome - thus identifying the "nonfunctioning' tumor as a possible negative prognostic factor (Madeira et al. 1998, Phan et al. 1998, Soreide et al. 2000, Rindi \& Bordi 2003) - albeit this finding was not confirmed by multivariate analysis. This discrepancy could also be due to the features of the patients enrolled in the present study, which includes both PETs and GI carcinoids. In fact, although the absence of an associated syndrome appears to be predictive of a negative prognosis in pancreatic tumors (Madeira et al. 1998, Phan et al. 1998) since the absence of specific symptoms could explain the late diagnosis, in GI carcinoids the lack of symptoms related to hormone overproduction could be due to a very small endocrine tumor that has not yet developed the carcinoid syndrome that is related to the presence of advanced disease. This is probably the case in the subgroup of patients with tumors located in the appendix $(n=8)$ or rectum $(n=13)$, since these are often detected at early stage due to the onset of an appendicitis-like syndrome or to an incidental finding during lower GI tract endoscopy respectively. However, $14.3 \%$ of these have been included in groups III to IV, confirming that - although less frequently advanced disease may already be present also in these patients, as suggested by the poor outcome of one of our patients with an appendiceal tumor who had advanced disease at time of diagnoses and died of disease at 81 months follow-up. Finally, in order to exclude a potential bias related to the small group of appendiceal tumors, we re-performed the multivariate analysis excluding such cases and confirmed the negative prognostic role of the pancreatic site, the poor degree of tumor differentiation, and the presence of distant extra-hepatic metastases. However, even concerning PETs, the role of the 'functional status' is not clear, as confirmed by the study of Hochwald et al. (2002), who observed similar survival rates irrespective of the presence of a specific associated syndrome. When analyzed according to the size of the primary tumor, the Ki67 value and degree of differentiation of the tumor cells, the present study confirmed that - as reported in the literature (La Rosa et al. 1996, Madeira et al. 1998, Rigaud et al. 2001, Furlan et al. 2004) these variables are clearly associated with a worse prognosis (Tables 2 and 5). However, some of these variables were not found to be significant by multivariate analysis, probably due to the fact that the total number of complete survival times observed in this study is too small to detect the simultaneous effects of 
combinations of factors. As far as the presence of sstr on tumor cells is concerned, we observed that the vast majority of our patients $(92.4 \%)$ had a positive SRS and that there was no difference in survival between patients with or without sstr expression. In addition, we observed that $75 \%$ of sstr-negative tumors were in the pancreas, and that all but one were not associated with a clinical syndrome. To our knowledge, the role of sstr as a prognostic factor has not previously been investigated and thus our findings can not be compared with those of other series. However, patients with a positive SRS might be expected to have a better prognosis since the presence of sstr on tumor cells allows the possibility of visualizing tumor lesions by SRS, thus making the tumor amenable to successful treatment either with unlabeled or labeled somatostatin analogs (Gibril \& Jensen 2004). However, certain elements need to be considered. In fact, since sstr are expressed in at least $80 \%$ of GEP ETs (Corleto et al. 2004), the number of patients with negative SRS is extremely low; thus a comparison between the two subgroups is difficult to perform. Furthermore, the role of this variable in modifying the survival of patients is probably influenced by the use of somatostatin analogs, which, in the present study, were administered in $96.4 \%$ of patients who underwent medical treatment. Thus, it is difficult to determine the real impact of the presence of sstr on the survival of patients with GEP ETs. The present study harbors some pitfalls. In fact, although prospective, the study design is affected by the long period of time over which it was performed, i.e. some 20 years. This must be taken into account when analyzing our findings since during the last two decades much progress has been made in our knowledge of GEP ETs, particularly in terms of diagnostic tools and therapeutic approach. An additional limitation related to the long duration of the study is that some of the variables under evaluation, such as Ki67 value and SRS findings, were not available in some patients enrolled during the earlier part of the study; thus we cannot exclude some influence on the prognostic significance of these variables. Furthermore, the enrolled population of the present study differs from other GEP ET epidemiological series due to the low number of appendiceal carcinoids $(n=8)$ and insulinomas $(n=1)$. The consecutive referral to a gastroenterology unit is likely to account for this difference. In fact, in clinical practice, most appendiceal carcinoids are accidentally diagnosed by surgeons, while most insulinomas are referred to endocrinologists for their specific syndrome.

The median length of our follow-up, i.e. 42.5 months, is in agreement with the follow-up value reported in other studies (Burke et al. 1997, Madeira et al. 1998, Chu et al. 2002, Hochwald et al. 2002, Furlan et al. 2004). This value is influenced by several elements, such as the inclusion of patients with recent diagnosis of GEP ET and the finding that most deaths occur at a short interval of time from diagnosis (median 18 months).

In conclusion this study reveals that, although considered heterogeneous as far as clinical, histological and biological features are concerned, GEP ETs show a similar frequency of metastases at the time of diagnosis, irrespective of the presence of an associated syndrome, and of the pancreatic or GI site of the primary tumor. Furthermore, since some features such as the good degree of differentiation and the expression of sstr - are present in the majority of these tumors, it is important to take these findings into consideration when planning medical treatment; in fact, this treatment usually consists of: somatostatin analogs (based on the presence of sstr) alone, or in association with interferon; and systemic chemotherapy, the reference treatment in poorly differentiated tumors (O'Toole et al. 2004).

In addition to the pancreatic site of the primary tumor, the most important negative prognostic factors are the poor degree of tumor cell differentiation and the presence of distant (particularly extra-hepatic) metastases; therefore, these factors should always be considered during disease staging, in order to identify those patients at higher risk and thus requiring more specific treatment and follow-up programs.

\section{Acknowledgements}

The authors are grateful to Mrs Marian Shields for help with the English.

\section{Funding}

This work was supported by a grant (2003063877_002) from the Italian Ministry for the University (MIUR). The authors declare that there is no conflict of interest that would prejudice the impartiality of this scientific work.

\section{References}

Barakat MT, Meeran K, Bloom \& SR 2004 Neuroendocrine tumors. Endocrine-Related Cancer 11 1-18.

Burke AP, Thomas RM, Elsayed AM \& Sobin LH 1997 Carcinoids of the jejunum and ileum. Cancer 79 1086-1093.

Chu QD, Hill HC, Douglass HO, Driscoll D, Smith JL, Nava HR \& Gibbs JF 2002 Predictive factors associated 
with long-term survival in patients with neuroendocrine tumors of the pancreas. Annals of Surgical Oncology 9 885-862.

Corleto VD, Scopinaro F, Angeletti S, Materia A, Basso N, Polettini E, Annibale B, Schillaci O, D’Ambra G, Marignani M, Gualdi G, Bordi C, Passaro EJ \& Delle Fave G 1996 Somatostatin receptor localization of pancreatic endocrine tumors. World Journal of Surgery 20 241-244.

Corleto VD, Nasoni S, Panzuto F, Cassetta S \& Delle Fave G 2004 Somatostatin receptors subtypes: basic pharmacology and tissue distribution. Digestive and Liver Disease 36 S8-S16.

Fishbeyn VA, Norton JA, Benya RV, Pisegna JR, Venzon DJ, Metz DC \& Jensen RT 1993 Assessment and prediction of long-term cure in patients with Zollinger-Ellison syndrome: the best approach. Annals of Internal Medicine 119 199-206.

Frucht H, Howard JM, Slaff JI, Wank SA, McCarthy DM, Malton PN, Vinayek R, Gardner JD \& Jensen RT 1989 Secretin and calcium provocative tests in the Zollinger Ellison syndrome: a prospective study. Annals of Internal Medicine 111 713-722.

Furlan D, Cerutti L, Uccella S, La Rosa S, Rigoli E, Genasetti A \& Capella C 2004 Different molecular profiles characterize well-differentiated endocrine tumors and poorly differentiated endocrine carcinomas of the gastroenteropancreatic tract. Clinical Cancer Research 10 947-957.

Gibril F \& Jensen RT 2004 Diagnostic uses of radiolabelled somatostatin receptor analogues in gastroenteropancreatic endocrine tumors. Digestive and Liver Disease 36 S106-S120.

Gullo L, Migliori M, Falconi M, Pederzoli P, Bettini R, Casadei R, Delle Fave G, Ceccarelli C, Santini D \& Tomassetti P 2003 Nonfunctioning pancreatic endocrine tumors: a multicentric clinical study. American Journal of Gastroenterology 98 2435-2439.

Hochwald SN, Zee S, Conlon KC, Colleoni R, Louie O, Brennan MF \& Klimstra DS 2002 Prognostic factors in pancreatic endocrine neoplasms: an analysis of 136 cases with a proposal for low-grade and intermediate-grade groups. Journal of Clinical Oncology 20 2633-2642.

Johanson V, Tisell LE, Olbe L, Wangberg B, Nilsson O \& Ahlman H 1999 Comparison of survival between malignant neuroendocrine tumors of midgut and pancreatic origin. British Journal of Cancer $\mathbf{8 0}$ 1259-1261.

Kent RB, van Heerden JA \& Wheiland LH 1981 Nonfunctioning islet cell tumors. Annals of Surgery 193 185-190.

La Rosa S, Sessa F, Capella C, Riva C, Leone BE, Klersy C, Rindi G \& Solcia E 1996 Prognostic criteria in nonfunctioning pancreatic endocrine tumors. Virchows Archives 429 323-333.

Lepage C, Bouvier AM, Phelip JM, Hatem C, Vernet C \& Faivre J 2004 Incidence and management of malignant digestive endocrine tumors in a well defined French population. Gut $\mathbf{5 3} 549-553$.

Madeira I, Terris B, Voss M, Denys A, Sauvanet A, Flejou JF, Vilgrain V, Belghiti J, Bernades P \& Ruszniewski P 1998 Prognostic factors in patients with endocrine tumors of the duodenopancreatic area. Gut $\mathbf{4 3}$ 422-427.

Modlin IM, Lye KD \& Kidd M 2003 A 5-decade analysis of 13715 carcinoid tumors. Cancer 97 934-959.

O’Toole D, Hentic O, Corcos O \& Ruszniewski P 2004 Chemotherapy for gastro-entero-pancreatic endocrine tumours. Neuroendocrinology 80 (Suppl 1) 79-84.

Panzuto F, Falconi M, Nasoni S, Angeletti S, Moretti A, Bezzi M, Gualdi G, Polettini E, Sciuto R, Festa A, Scopinaro F, Corleto VD, Bordi C, Pederzoli P \& Delle Fave G 2003 Staging of digestive endocrine tumors using helical computer tomography and somatostatin receptor scintigraphy. Annals of Oncology 14 586-591.

Panzuto F, Severi C, Cannizzaro R, Falconi M, Angeletti S, Pasquali A, Corleto VD, Annibale B, Buonadonna A, Pederzoli P et al. 2004 Utility of combined use of plasma levels of chromogranin A and pancreatic polypeptide in the diagnosis of gastrointestinal and pancreatic endocrine tumors. Journal of Endocrinological Investigation 27 6-11.

Pape UF, Bohmig M, Berndt U, Tiling N, Wiedenmann B \& Plockinger U 2004 Survival and clinical outcome of patients with neuroendocrine tumors of the gastroenteropancreatic tract in a German referral center. Annals of the New York Academy of Sciences $\mathbf{1 0 1 4}$ 222-233.

Phan GQ, Yeo CJ, Hruban RH, Lillemoe KD, Pitt HA \& Cameron JL 1998 Surgical experience with pancreatic neuroendocrine tumors: review of 125 patients. Journal of Gastrointestinal Surgery 2 473-482.

Quaedvlieg PF, Visser O, Lamers CB, Janssen-Heijen ML \& Taal BG 2001 Epidemiology and survival in patients with carcinoid disease in the Netherlands. Annals of Oncology 12 1295-1300.

Rigaud G, Missiaglia E, Moore PS, Zamboni G, Falconi M, Talamini G, Pesci A, Baron A, Lissandrini D, Rindi G, Grigolato P, Pederzoli P \& Scarpa A 2001 High resolution allelotype of nonfunctional pancreatic endocrine tumors: identification of two molecular subgroups with clinical implications. Cancer Research 61 285-292.

Rindi G \& Bordi C 2003 Highlights on biology of endocrine tumors of the gut and pancreas. Endocrine-Related Cancer 10 427-436.

Shebani KO, Souba WW, Finkelstein DM, Stark PC, Elgadi KM, Tanabe KK \& Ott MJ 1999 Prognosis and survival in patients with gastrointestinal tract carcinoid tumors. Annals of Surgery 229 815-823.

Soreide JA, van Heerden JA, Thompson GB, Schleck C, Ilstrup DM \& Churchward M 2000 Gastrointestinal carcinoid tumors: long-term prognosis for surgically treated patients. World Journal of Surgery 24 1431-1436.

Sutton R, Doran HE, Williams EMI, Vora J, Vinjamuri S, Evans J, Campbell R, Raraty MGT, Ghaneh P, Hartley 
M, Poston GJ \& Neoptolemos JP 2003 Surgery for midgut carcinoid. Endocrine-Related Cancer 10 469-481.

Taal BG \& Visser O 2004 Epidemiology of neuroendocrine tumors. Neuroendocrinology 80 (Suppl 1) 3-7.

Weber HC, Venzon DJ, Lin JT, Fishbein VA, Orbuch M, Strader DB, Gibril F, Metz DC, Fraker DL, Norton JA et al. 1995 Determinants of metastatic rate and survival in patients with Zollinger-Ellison syndrome: a prospective long-term study. Gastroenterology 108 1637-1649.

Yu F, Venzon DJ, Serrano J, Goebel SU, Doppman JL, Gibril F \& Jensen RT 1999 Prospective study of the clinical course, prognostic factors, causes of death, and survival in patients with long-standing Zollinger-Ellison syndrome. Journal of Clinical Oncology 17 615-630. 